Pineda-Alfonso, José A. (2015). Educar para la ciudadanía trabajando con temas controvertidos en Educación Secundaria Obligatoria. Revista de Investigación Educativa, 33(2), 353-367.

DOI: http://dx.doi.org/10.6018/rie.33.2.208441

\title{
Educar para la ciudadanía trabajando con temas controvertidos en Educación Secundaria Obligatoria
}

\section{Educating for citizenship working with controversial issues in Compulsory Secondary Education}

\author{
José A. Pineda-Alfonso \\ Departamento de Didáctica de las Ciencias Experimentales y Sociales. Facultad de Ciencias de la Educación \\ Universidad de Sevilla
}

\begin{abstract}
Resumen
Presentamos una investigación basada en una propuesta de trabajo con temas controvertidos que pretende una reorientación de la enseñanza de las ciencias sociales para dar respuesta a una demanda: la necesidad de adecuar la escuela a las necesidades del mundo en el que vivimos como forma de promover una ciudadanía más participativa. Esta propuesta curricular ha sido experimentada con un grupo de alumnos de $4^{\underline{0}}$ curso de Educación Secundaria Obligatoria y de su seguimiento investigativo se deriva la posibilidad de desarrollar aprendizajes procedimentales relacionados con la identificación de problemas, la planificación de su resolución, la argumentación, la producción de conclusiones con sentido personal y la reflexión sobre el propio proceso de aprendizaje.

Palabras clave: ciudadanía, participación, temas controvertidos, aprendizaje procedimental.
\end{abstract}

Correspondencia: C/ Pirotecnia s/n. 41013 Sevilla. Teléfono: 955420 785. FAX: 95.455.17.33. E-mail: apineda@us.es Este trabajo es resultado parcial del Proyecto I+D, con referencia EDU2011-23213, financiado por el Ministerio de Ciencia e Innovación del Gobierno de España y por Fondos FEDER, titulado "Estrategias de formación del profesorado para educar en la participación ciudadana". 


\begin{abstract}
We present a research-based project work with controversial issues that seeks a reorientation of social science education to meet demand: the need to bring the school to the needs of the world in which we live as a way of promoting a more participatory citizenship. This proposed curriculum has been experienced with a group of students in the $4^{\text {th }}$ year of compulsory secondary education and follow-up research is derived from the ability to develop learning procedural great educational value related to problem identification, planning your resolution, argumentation, production conclusions with personal meaning and reflection on the learning process.
\end{abstract}

Keywords: citizenship, participation, controversial issues, procedural learning.

\title{
El trabajo con problemas en la enseñanza de las ciencias sociales
}

En los últimos años parece abrirse paso la idea de que la escuela permanece ajena a las demandas intelectuales que exige el tratamiento de los problemas sociales y ambientales de nuestro tiempo, pues las metodologías de trabajo, básicamente transmisivas y repetitivas no desarrollan el pensamiento complejo y no capacitan para comprender las conexiones entre los temas estudiados y los problemas contemporáneos (García y De Alba, 2008). En concreto, investigaciones recientes han puesto de manifiesto que la manera tradicional de enseñar Ciencias Sociales en España no supone un aprendizaje útil para la formación de ciudadanos (Navarro y De Alba, 2011). En este sentido algunos autores señalan la necesidad de enseñar a los alumnos estrategias para manejarse con la saturación de información de la sociedad del conocimiento, para conseguir autonomía en la planificación y toma de decisiones, y para lograr una mayor capacidad de reflexión sobre el propio proceso de aprendizaje (Monereo, 2001).

Una vía para la búsqueda de la conexión entre los contenidos curriculares y los problemas sociales ha sido el enfoque de trabajo sobre temas controvertidos de la actualidad. Este nuevo interés sobre el tratamiento de problemas sociales relevantes surgió del convencimiento de que la enseñanza de las Ciencias Sociales debe estar orientada a la comprensión de la realidad social y sus problemas, a formar el pensamiento social para gestionar la complejidad de esta realidad, y a favorecer la participación para la construcción de la democracia y la mejora de la convivencia (Canal, Costa y Santisteban, 2012). Estas finalidades, en opinión de muchos autores, son las más importantes que pueden plantearse en la educación (Parker, 2008).

Hay un cuerpo considerable de literatura acerca de las ventajas que la incorporación de cuestiones controvertidas en el currículo escolar tiene en cuanto al desarrollo de habilidades relacionadas con la investigación, tales como la capacidad para recopilar e interpretar datos y comprender la naturaleza de las pruebas, la planificación de acciones, la identificación de relaciones causales y la distinción entre causalidad y correlación, y la elaboración de teorías y conclusiones usando el pensamiento creativo (Young, Commins y Kington, 2002).

Además, la inclusión de temas controvertidos en el aula permite el aprendizaje participativo promoviendo la escucha activa de diversos puntos de vista, capacitando 
para expresar y cambiar las opiniones a la luz de los nuevos argumentos e informaciones. Junto a los argumentos éticos y cívicos, y las razones ligadas al desarrollo de capacidades cognitivas, se esgrime también como argumento para basar el currículum en el trabajo con problemas el desarrollo del razonamiento y de la reflexión sobre el propio conocimiento como construcción social (Jiménez-Aleixandre y Erduran, 2008). La argumentación y la discusión permite a los alumnos ser reflexivos y críticos en relación con las cuestiones controvertidas, y la controversia permite la interacción entre Ciencia y Sociedad (Alpha Nielsen, 2009). De hecho, el espíritu de renovación que la educación para la ciudadanía ha introducido en los sistemas educativos de muchos países ha contribuido a recuperar el interés por el desarrollo en los alumnos de la capacidad de argumentación (Jiménez-Aleixandre y Erduran, 2008) y de tomar decisiones sobre problemas y controversias socio-científicas (Hermann, 2008); un enfoque que ha sido específicamente desarrollado, desde las ciencias, por la línea de la alfabetización científica y ciudadana (González y Correa, 2006; Gil Pérez y Vilches, 2006). Y desde el campo de las Ciencias Sociales por el intento de poner la enseñanza de la Historia al servicio de la educación para la ciudadanía (Seixas, P. \&Peck, C., 2004; Clark, P., 2013).

Junto a esto, también hay evidencias de que la controversia y el desacuerdo desarrolla la "reflexión inteligente" (Millar, 1997) en un mundo de complejidad creciente. El interés por el fomento del pensamiento crítico y reflexivo se inscribe en el nuevo paradigma de enseñanza centrada en el alumno y en la autorregulación de su propio proceso de aprendizaje (self regulated learning) como estrategia para "aprender a aprender", desarrollando mayor autonomía, responsabilidad y control sobre el propio aprendizaje (González Cascón et al., 2010). El trabajo con problemas ha sido puesto en relación con el desarrollo de la metacognición, pues permite relacionar los conocimientos previos con las conclusiones y reflexionar sobre las propias dificultades y maneras personales de aprender. La capacidad para reflexionar sobre las propias acciones se ha denominado en Psicología del Desarrollo "metaconocimiento" y es una de las manifestaciones de la conciencia, del darse cuenta, en este caso de que se está aprendiendo algo (Delval, 1994).

Todo este cuerpo de evidencias ha hecho que, a iniciativa de numerosos organismos internacionales, los planes de estudio de distintos países pusieran en marcha programas de ciudadanía que reconocen que los conocimientos y habilidades que se enseñan en la escuela están divorciados de las situaciones de la vida cotidiana de los jóvenes y de los problemas complejos del mundo moderno (Young, Commins y Kington, 2002). Por ejemplo, en el sistema escolar danés se recoge como una competencia a desarrollar el fomento de la argumentación y de la toma de decisiones socio-científicas (Danish Ministry of Education, 2008). Y en el currículum de Reino Unido la enseñanza de la Ciudadanía incluye la controversia, y se insta a enseñar a los alumnos la manera de recopilar, analizar e interpretar los datos para entender sus limitaciones, validez y fiabilidad, y para conocer la relación entre datos, evidencias, teorías y explicaciones (Qualifications and Curriculum Authority, 2006). Todas estas evidencias llevaron a algunos autores a afirmar que cualquier escuela o institución educativa que evite intencionadamente las cuestiones controvertidas podría estar incurriendo en una negligencia o en una práctica inadecuada (Stradling, 1984). 


\section{El diseño metodológico de la investigación}

Ubicándonos en el marco de trabajo que acabamos de esbozar, hemos realizado una investigación, basada en la experimentación de una propuesta curricular para Ciencias Sociales de $4^{\circ}$ curso de Educación Secundaria Obligatoria, siguiendo el formato de una investigación-acción y el modelo clásico de profesor-investigador, aunque incorporando determinados elementos y técnicas más propios del paradigma racionalista, como el establecimiento de un sistema de categorías o el análisis cuantitativo de los resultados (Heiman, 2011). Este planteamiento es coherente con una larga tradición de investigación para mejorar la propia práctica en la educación secundaria, y del que tenemos algunas experiencias recientes (McCoy, 2013; Oolbekkink-Marchand, van der Steen \& Nijveld, 2014).

1) Hemos partido de la identificación de un aspecto problemático de la realidad del aula que queríamos comprender para mejorar. Se trataba de los escasos resultados que se obtenían en los aprendizajes en la dimensión procedimental (ADP), pues a menudo quedan relegados en el currículum, y en la práctica mayoritaria, en favor de los aprendizajes conceptuales.

2) El segundo paso consistió en el diseño de una propuesta o plan de actuación que tomó la forma de un Ámbito de Investigación Escolar (AIE) ${ }^{1}$. En el marco teórico del Proyecto IRES ${ }^{2}$ este es un enfoque que permite seleccionar y organizar los contenidos curriculares en torno a problemáticas sociales y ambientales, en este caso relacionadas con el "conflicto y la convivencia" (Pineda-Alfonso y García-Pérez, 2011). Consideramos que "el conflicto y la convivencia" configuran una problemática controvertida de gran interés para la educación ciudadana, y permite el desarrollo de aprendizajes procedimentales relacionados con el planteamiento y resolución de problemas, con el trabajo con la argumentación y la discrepancia en la interacción en grupo y con la reflexión sobre el propio proceso de aprendizaje.

Nuestro enfoque se ha basado en el planteamiento de problemas de investigación por parte de los alumnos con ayuda del profesor, seguido de una fase de exploración y expresión de las concepciones sobre la problemática de la convivencia, elaboración de hipótesis provisionales, interacción y contraste de éstas con nuevas informaciones, y elaboración de conclusiones para ser comunicadas al resto del grupo-clase. El trabajo en grupo, como dimensión de una ciudadanía cooperativa, lo hemos considerado una parte fundamental de la propuesta. Como indican algunos estudios empíricos, la interacción comunicativa y la "controversia constructiva" que se da en el grupo de trabajo, no sólo tienen un efecto positivo sobre el desarrollo de competencias sociales sino que también estimulan el esfuerzo de los estudiantes y mejoran el razonamiento cognoscitivo y la comprensión de los contenidos básicos de las temáticas que se estudian (Johnson y Johnson, 2009).

1 Desde la perspectiva de IRES, los “ámbitos de investigación escolar" son conjuntos de problemas socioambientales que actúan como organizadores del currículo, son relevantes para la comprensión de la realidad e integran, definen y concretan el conocimiento escolar deseable.

2 El Proyecto IRES (Investigación y Renovación Escolar) es un programa de investigación y acción educativa, en cuyo marco se han desarrollado, desde 1991, un número importante de investigaciones educativas y de experiencias de innovación didáctica. El IRES constituye, asimismo, un marco para el desarrollo profesional de los docentes. 
3) Este AIE fue experimentado durante el curso 2010-11 con un grupo de alumnos de $4^{\mathrm{o}}$ de ESO $(\mathrm{n}=21)$ de un Instituto de Educación Secundaria Obligatoria. Pretendíamos investigar el impacto que esta propuesta podía tener en cuanto a la mejora en los resultados de los aprendizajes procedimentales de nuestros alumnos. En este sentido, el problema de investigación formulado sería: ¿qué procesos de aprendizaje procedimental se dan en nuestros alumnos cuando se desarrolla un AIE sobre el conflicto y la convivencia con una metodología didáctica de trabajo en torno a problemas?

Para dar respuesta a esta cuestión hemos registrado las producciones de los alumnos y las dinámicas e interacciones en el aula a través de un proceso de observación participante, de larga tradición sobre todo en estudios etnográficos (Cohen, Manion \& Morrison, 2011). Los instrumentos utilizados para ello han sido: los cuadernos de clase de los alumnos (F1), las grabaciones en audio de uno de los grupos de debate (F2), y el Diario del Investigador (F3).

4) La información procedente de los documentos escritos y de las transcripciones de audio fue tratada según técnicas de "análisis del contenido" (Krippendorff, 2013) para ser codificadas y convertidas en unidades de información utilizando un sistema de categorías con tres valores (ADP1, ADP2, ADP3) (Tabla 1), y siguiendo una hipótesis de progresión de menor a mayor complejidad. Dentro del Proyecto IRES, las hipótesis de progresión son una propuesta relativa a la posible evolución en la construcción del conocimiento de los alumnos sobre una determinada temática, de tal manera que es posible establecer distintos niveles de formulación de este conocimiento siguiendo una progresión gradual del mismo (García, 1998). En lo que respecta a los aprendizajes procedimentales, estas categorías han sido:

1) La capacidad procedimental para trabajar con problemas: identificar y plantear problemas -pues supone un ejercicio de abstracción y generalización-, proponer hipótesis explicativas, planificar su resolución, manejar y tratar distintas fuentes de información en distintos formatos, construir ideas y argumentos y contrastarlos en el debate gestionando la discrepancia, y finalmente proponer conclusiones fruto de la elaboración personal. Esta problematización del conocimiento supone además un cambio profundo en la concepción del saber, que pasa de ser considerado una mercancía que otorga status y poder a una forma de atribuir sentido a nuestra realidad para comprenderla y participar, como ciudadanos, en su transformación y mejora.

2) La reflexión sobre el propio proceso de aprendizaje es otro metaprocedimiento de gran valor educativo, estrechamente vinculado con la metacognición y con la competencia básica de "aprender a aprender". Algunas de las características de la metacognición que han sido destacadas han sido: la determinación de objetivos, la auto-observación y la autorregulación que permiten la revisión y el ajuste de la propia actuación, la decisión con respecto a las estrategias a emplear, la planificación y el desarrollo de la actuación, la corrección de posibles desviaciones y la evaluación de la tarea realizada (Siegler \& Richard, 1989).

Las unidades de información han sido tratadas en su progresión longitudinal dividiendo el curso en tres momentos para la recogida de datos (correspondientes a las tres unidades didácticas del proyecto, denominadas UI, U2 y U3) y dentro de cada uno de ellas hemos seleccionado una serie de actividades que considerábamos con 
potencialidad para ofrecernos informaciones relevantes para nuestra investigación: una actividad inicial de planteamiento de problemas de investigación, una actividad de planificación de la investigación, una actividad de conclusión individual de la investigación, una actividad de debate y discusión y una actividad de reflexión sobre el propio proceso de aprendizaje y sus condicionantes.

\section{Tabla 1}

Sistema de categorías e hipótesis de progresión

\begin{tabular}{|c|c|c|}
\hline ADP1 & ADP2 & ADP3 \\
\hline $\begin{array}{l}\text { - Escasa capacidad para } \\
\text { plantear problemas que } \\
\text { permitan abrir un proceso } \\
\text { de investigación escolar }\end{array}$ & $\begin{array}{l}\text { - Formulación de proble- } \\
\text { mas de investigación des- } \\
\text { criptivos, con escaso grado } \\
\text { de abstracción y generali- } \\
\text { zación }\end{array}$ & $\begin{array}{l}\text { - Formulación de proble- } \\
\text { mas de investigación con- } \\
\text { trovertidos con un mayor } \\
\text { grado de abstracción y ge- } \\
\text { neralización }\end{array}$ \\
\hline $\begin{array}{l}\text { - La planificación se identi- } \\
\text { fica con la búsqueda de in- } \\
\text { formación }\end{array}$ & $\begin{array}{l}\text { - La planificación tiene en } \\
\text { cuenta las distintas fases } \\
\text { del proceso de investiga- } \\
\text { ción y su utilidad }\end{array}$ & $\begin{array}{l}\text { - La planificación sigue un } \\
\text { proceso flexible y dinámi- } \\
\text { co, y su finalidad es la re- } \\
\text { solución de los problemas } \\
\text { planteados }\end{array}$ \\
\hline $\begin{array}{l}\text { - La copia y la repetición sus- } \\
\text { tituye a la elaboración perso- } \\
\text { nal y a la argumentación }\end{array}$ & $\begin{array}{l}\text { - Diálogo espontáneo, uti- } \\
\text { lización de informaciones } \\
\text { escritas para construir opi- } \\
\text { nión argumentada y mayor } \\
\text { grado de simbolización }\end{array}$ & $\begin{array}{l}\text { - Trabajo colaborativo en el } \\
\text { grupo de debate }\end{array}$ \\
\hline $\begin{array}{l}\text { - La reflexión se limita a la } \\
\text { queja por las dificultades } \\
\text { encontradas y a la observa- } \\
\text { ción de lo evidente y per- } \\
\text { ceptible del contexto }\end{array}$ & $\begin{array}{l}\text { - Reflexión centrada en la } \\
\text { pertinencia y novedad de } \\
\text { las informaciones, recursos } \\
\text { y nuevas tareas. Sin refe- } \\
\text { rencias todavía a la meto- } \\
\text { dología o al papel del pro- } \\
\text { fesor y de los alumnos en el } \\
\text { proceso }\end{array}$ & $\begin{array}{l}\text { - Reflexión sobre cómo dis- } \\
\text { tintos factores del contexto, } \\
\text { de la organización y de las } \\
\text { tareas en la escuela se vincu- } \\
\text { lan con la convivencia y con } \\
\text { su proceso de aprendizaje. }\end{array}$ \\
\hline
\end{tabular}

\section{Elaboración propia.}

Finalmente, las 2.903 unidades de información que resultaron de la codificación fueron gestionadas con el programa Atlas.Ti, a fin de obtener una clasificación de las mismas para dar respuesta al problema investigado. 


\section{Resultados y discusión}

Vamos a centrar la descripción de los resultados de la investigación en la dimensión de los aprendizajes procedimentales, pues consideramos -como dijimos- que el desarrollo de unos procedimientos básicos -a modo de "metaprocedimientos"- constituye un aprendizaje de gran valor desde el punto de vista de la educación para la ciudadanía participativa.

En este sentido, observamos en las tablas (Tabla 2 y 3) y en el gráfico adjunto (Gráfico 1) que en el momento inicial de la intervención (U1) hay un claro predominio (73.57\%) de las unidades de información de valor 1 (ADP1), el más cercano al repertorio de habilidades procedimentales de un menor nivel de complejidad. Paralelamente, observamos la ausencia de unidades de información de valor 3 (ADP3), el de mayor nivel de complejidad e incompatible con las conductas y pautas de acción mayoritarias en la escuela. En el momento subsiguiente (U2) observamos una evolución gradual de los aprendizajes procedimentales con un incremento significativo (50\%) de las unidades de información de valor 2 (ADP2), la aparición de un porcentaje todavía bajo $(1.56 \%)$ de unidades de valor 3 (ADP3)y la persistencia de un porcentaje aún considerable (48.43\%) de unidades de valor 1 (ADP1). Por último, en el momento final de la intervención (U3) tenemos un predominio (49.16\%) de las unidades de valor 3 (ADP3), aunque se muestran pervivencias de valores anteriores (ADP1 y ADP2) (15.08\% y 35.75\%). En resumen, pensamos que la gráfica muestra evidencias de la posibilidad del enriquecimiento y complejización de los aprendizajes procedimentales de nuestros alumnos, sin bien, la evolución es gradual y dinámica y está marcada por avances, retrocesos y estancamientos.

Tabla 2

Unidades de información ADP en valores absolutos

\begin{tabular}{|c|c|c|c|}
\hline & U1 & U2 & U3 \\
\hline ADP1 & 181 & 155 & 27 \\
\hline ADP2 & 65 & 160 & 64 \\
\hline ADP3 & 0 & 5 & 88 \\
\hline TOTAL & 246 & 320 & 179 \\
\hline
\end{tabular}

Tabla 3

Unidades de información ADP en valores porcentuales

\begin{tabular}{|c|c|c|c|}
\hline & U1 & U2 & U3 \\
\hline ADP1 & $73,57 \%$ & $48,43 \%$ & $15,08 \%$ \\
\hline ADP2 & $26,42 \%$ & $50 \%$ & $35,75 \%$ \\
\hline ADP3 & $0,00 \%$ & $1,56 \%$ & $49,16 \%$ \\
\hline
\end{tabular}




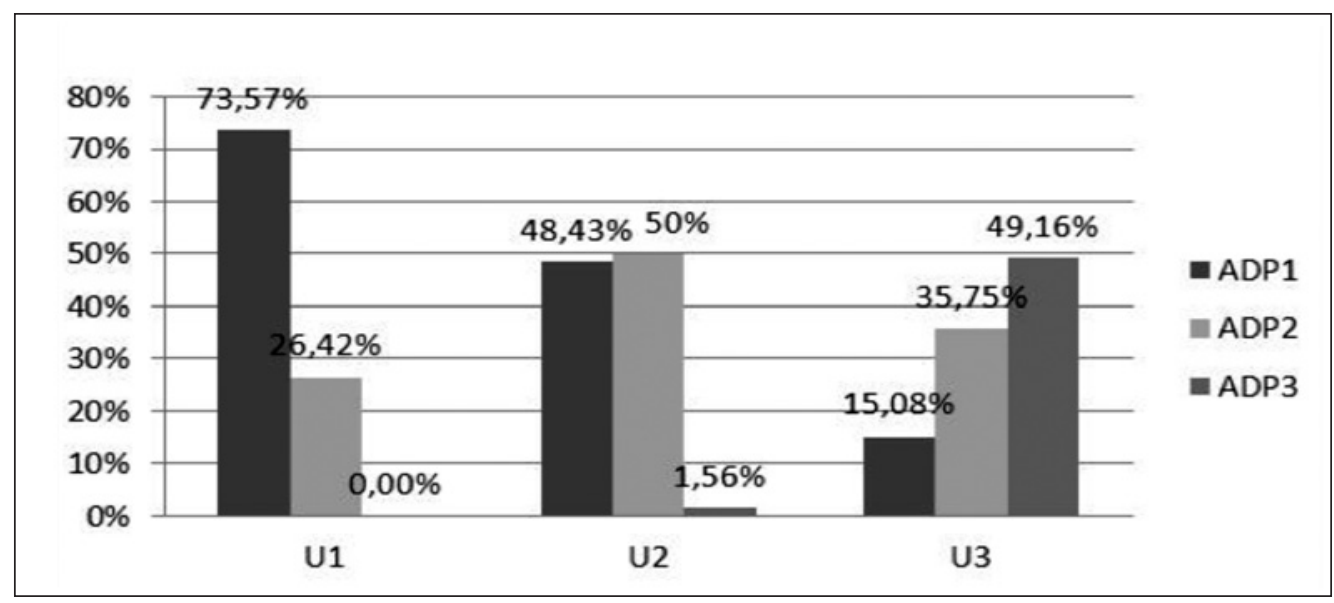

Gráfico 1. Distribución longitudinal de los aprendizajes procedimentales. Elaboración propia.

\section{El estado inicial de los aprendizajes procedimentales}

El enfoque del trabajo con problemas se enfrenta, como obstáculos fundamentales de parte de los alumnos, con la tendencia a copiar y memorizar sin comprensión, que hacen muy difícil la argumentación y la elaboración personal; y con una concepción del conocimiento escolar como producto acabado, que proviene de otro (de quien se aprende), que no tiene ninguna utilidad ni responde a objetivos relacionados con el aprendiz, sino simplemente a los requerimientos y expectativas de la cultura escolar, y que muestra un gran arraigo desde su constitución formal en el siglo XIX, como nos han mostrado las investigaciones sobre la sociogénesis de las materias escolares (Goodson, 2000).

En coherencia con esto, en el estado inicial de nuestra intervención, valor 1 de nuestra hipótesis de progresión (ADP1), nuestros alumnos no le encontraban sentido ni utilidad al trabajo con problemas, más bien se sentían desconcertados por la responsabilidad y el esfuerzo de cambio que se les pedía, pues había que resituarse en un nuevo marco de relaciones en el aula. En efecto, la cultura escolar mayoritaria fomenta algo más cómodo: esperar la "respuesta verdadera" que viene del profesor y repetir tareas mecánicas sin sentido personal, esto crea adherencias y servidumbres y termina por instalarse de manera permanente en el repertorio de conductas de los alumnos.

En este sentido las propuestas de problemas de investigación de nuestros alumnos eran tan generales que no presentaban contornos y límites definidos, o bien, en el extremo opuesto, se basaban en una casuística tan concreta que resultaba irrelevante. Otras veces simplemente entrañaban alguna queja o dilema moral pero tampoco permitían abrir un proceso de investigación sobre la temática planteada que permitiese la movilización de informaciones y la diversidad de respuestas argumentadas. En otros casos se planteaba una pregunta típica de las actividades escolares, que se resolvía adjuntando la respuesta verdadera que a menudo aparece en letra negrita en los libros de texto. 
[Formula algún problema de investigación relacionado con el conflicto y la convivencia a escala social]: A[lumno]: “¿Cómo se puede tener la sangre fría de tirar bombas nucleares sin llegar a pensar en la muerte de niños y personas?". [Cuaderno del alumno].

En cuanto a la planificación, en este estado inicial de las destrezas procedimentales de nuestros alumnos, estuvo centrada en la idea de redactar un informe o "trabajo" al uso, identificando el proceso exclusivamente con la búsqueda y transcripción de informaciones $y$, a menudo, planeando de manera mimética una secuencia de tareas similar a la que se suele realizar en clase.

[¿Cómo planificamos la investigación de estos problemas?]:

A: "Buscar información de cómo se resuelve un conflicto, proponer e inventar tipos de conflictos, representar distintos tipos de conflictos, buscar conflictos entre países". [Cuaderno del alumno].

Con respecto al tratamiento de la información, y a la capacidad para argumentar y elaborar conclusiones propias, habíamos pedido a nuestros alumnos que, utilizando las conclusiones escritas, parciales y finales, de los trabajos de investigación, establecieran un debate en grupo. $Y$, a pesar de las instrucciones de trabajo y de la insistencia del profesor, a menudo se limitaban a leer por turnos, dictando y repitiendo lo que habían escrito previamente en sus cuadernos de clase, mostrando cierta incapacidad para salir del circuito de la repetición y la copia y para acceder a la elaboración propia y a la capacidad para argumentar y construir opinión.

P[rofesor]: “Pero, un momento, no se trata de leer lo que cada uno tiene puesto. A1: No, si, si primero lo leemos pero luego lo ponemos en común. P: Ah, vale, vale (...) y luego lo debatís.

A2: Ya, ya, después nos ponemos de acuerdo". [Grabación de audio de un grupo de debate].

Por tanto, en este primer nivel de los aprendizajes procedimentales, y en coherencia con lo anterior, abundaron las conductas de repetición de lo escrito, o de adición de partes, en detrimento de su utilización en la dinámica del debate. Nuestros alumnos no contrastaban opiniones, entendían que debatir es sinónimo de "ponerse de acuerdo", pues la evitación del conflicto y la discrepancia es lo propio de la cultura escolar. En este sentido, mostraban un apego a la cultura escrita y a los "deberes" de clase que les impedía el diálogo fluido y la argumentación. Desde luego, tampoco concebían que tras la conclusión fuese posible ni pertinente formular nuevos problemas de investigación que permitiesen continuar el proceso, pues pensaban en un modelo de conclusión cerrada, fruto de un conocimiento acabado.

Cuando pedimos a nuestros alumnos que reflexionasen sobre la nueva manera de trabajar basada en el planteamiento y resolución de problemas, y sobre cómo construían sus ideas, observamos, en el valor 1 de la hipótesis de progresión, que no había reflexión sobre el propio proceso de aprendizaje, o bien ésta era muy simplificadora. 
A partir de aquí se progresaba hacia una reflexión incipiente que se centraba en lo más evidente: la pertinencia de las informaciones, la utilización de recursos nuevos más atractivos y la valoración de las nuevas tareas. Se recreaban en la novedad del método, en la oportunidad que brindaba para opinar, y se realizaban observaciones con respecto a los "fallos" que se cometían en la realización de las tareas; pero no había ninguna referencia a los contenidos, a la metodología, ni al nuevo papel que desempeñaba el profesor y ellos mismos en el proceso, ni a la relación de todo esto con los aprendizajes.

Con respecto a las reflexiones sobre la influencia del contexto escolar y sobre cómo las relaciones y las contingencias materiales y de espacio y tiempo influían en la convivencia y en los procesos de aprendizaje en la escuela, sólo alcanzaban a percibir lo evidente. Observaban la falta de espacios y se quejaban de la sobrecarga de los horarios y de otras deficiencias, pero no las vinculaban con los comportamientos de las personas ni con los objetivos de la institución escolar, y no las concebían como condicionantes de los aprendizajes y de las relaciones de convivencia.

\section{La progresión de los aprendizajes en la dimensión procedimental}

Creemos que el elemento clave en la progresión de los aprendizajes (valores ADP2 y ADP3 de nuestra hipótesis de progresión), fue la ruptura con el imaginario de las repeticiones, propiciado por la adopción, por parte del profesor, de un estilo menos explicativo y más "deconstructor", que a través de interrogantes cortaba las inercias e invitaba a la reflexión y a la construcción de la propia opinión:

A1: “El que tiene más (...) más (...) el que tiene ahí que mandar es el profesor (...) y siempre nos lo han dicho aquí que siempre que tengamos un conflicto vayamos al profesor.

P: ¿Eso es lo que creemos que es mejor? (...) [silencio]... eso es lo que nos han dicho aquí pero, ¿qué pensáis?, aquí entre nosotros, sin que se entere nadie. A4: que podríamos hacerlo nosotros mismos, ¿no? (...)”. [Grabación de audio de un grupo de debate]

Este tipo de intervenciones favorecía una cierta liberación de las ataduras del conformismo paralizante relacionado con el sesgo confirmatorio de las ideas -"lo que se hace aquí", "lo que nos han dicho que hagamos aquí", etc.-, y progresivamente permitía que el diálogo se hiciera más dinámico, fluido y espontáneo, y aparecieran ideas nuevas con las que cada uno alimentaba el proceso de argumentación.

Consideramos, pues, un elemento fundamental de la progresión, en esta dimensión del trabajo con problemas, el paso de la inercia de la repetición de tareas, siguiendo el formato de copia y de mínima interacción en los debates -trabajo aditivo sin contraste de ideas-, como forma de evitar los conflictos y la discrepancia, al diálogo espontáneo, utilizando las informaciones escritas para construir opinión argumentada y acceder a un mayor grado de simbolización.

En la medida en que esto ocurría, los debates se dinamizaban y el planteamiento de problemas comenzaba a tener sentido, pues éstos conectaban con intereses y curio- 
sidades sentidas por los alumnos, eran relevantes, permitían movilizar informaciones pertinentes para construir una respuesta que cuestionaba una parcela de la realidad o del saber, y daban cierto juego para iniciar un proceso de investigación abierto. De esta forma, cuando planteaban un dilema, éste apuntaba a una verdadera problemática que permitía formular hipótesis de trabajo y que podía tener distintas respuestas argumentadas. La progresión continuó con la formulación de problemas que ya no eran meramente descriptivos, no se trataba de indagar en las características de un fenómeno, sino que implicaban un mayor grado de abstracción y generalización, y además eran problemas controvertidos o candentes. En relación con el polémico tema de la energía nuclear:

[Formula algún problema de investigación relacionado con el conflicto y la convivencia a escala social]: A: “¿Por qué España no posee armas nucleares?, ¿sería mejor tenerlas? [Cuaderno del alumno]

La planificación tenía ahora en cuenta las distintas fases del proceso de investigación y su utilidad. La búsqueda de información ya no era el objetivo único, sino un elemento más, y su tratamiento una fase más, dentro de un proceso. Las hipótesis iniciales se entendían como una opinión que serviría, tras la conclusión final, para comparar y comprobar lo que habíamos aprendido. La planificación de la investigación incluía el trabajo colaborativo en equipo y las puestas en común en las que "cada uno encontraba algo diferente".

[Planificación]: A1: “Lo primero sería saber lo que vamos a estudiar; proponer preguntas que traten sobre el tema; responderlas sin conocimiento, con respuesta personal; buscar información sobre el tema; volver a responder las preguntas con conocimientos más avanzados, profundizar; averiguar lo que has aprendido del problema de investigación. Yo creo que lo de responder a las preguntas sin conocimientos (respuesta personal, lo que tú piensas en el momento de la pregunta) está muy bien porque cuando buscas información y adquieres conocimientos del tema te das cuenta de los errores que tenías y que no son como tu creías. Todos los pasos son importantes, buscar información es muy importante porque enriquece mucho nuestros conocimientos sobre el tema. Las hipótesis finales tienen que ser más elaboradas y complejas que las primeras y darse cuenta de lo aprendido en el problema de investigación". [Cuaderno del alumno].

El proceso de investigación ya no consistía en un listado de pasos a seguir sino que se constituía en un discurso con un hilo conductor que denotaba la comprensión del proceso de investigación e incluía la reflexión sobre lo que se aprende y cómo se aprende. La planificación seguía un proceso flexible y dinámico, comprendiendo realmente su sentido y utilidad y la finalidad última de la resolución de los problemas planteados, a los que se respondía en las conclusiones con la construcción de respuestas argumentadas con sentido personal. 
Con respecto a las reflexiones sobre el contexto escolar y su influencia en los procesos de aprendizaje, observamos un paso más en la progresión cuando realizamos actividades relacionadas con la investigación acerca de cómo se gestiona el conflicto y la convivencia en una organización, y concretamente en la institución escolar. Los alumnos tuvieron la oportunidad de reflexionar sobre cómo distintos factores del contexto, de la organización y de las tareas en la escuela, se vinculaban con la convivencia y con su proceso de aprendizaje.

Ya no se trataba de una mera queja por las deficiencias de las instalaciones, o por lo inadecuado de los espacios y los tiempos, ahora eran capaces de vincular los contextos espaciales y temporales con las relaciones entre las personas y los comportamientos, y esto suponía cierta reflexión sobre el propio proceso de aprendizaje. Cuando manejaban documentos para conocer la organización y los procedimientos de funcionamiento de su propia escuela tomaban conciencia de la diferencia entre lo declarativo y lo real, entre el contenido manifiesto de la institución y el contenido oculto o latente. Así pues, adoptaban una posición crítica, utilizando los conceptos aprendidos y tomando conciencia de la complejidad de las relaciones, analizando las entrevistas hechas a los profesores y reflexionando sobre las incoherencias entre los documentos organizativos y curriculares y los procedimientos y prácticas diarias.

Con respecto a esto, opinaban que los mecanismos de participación establecidos no funcionaban en la práctica, los derechos existían sólo en el papel y no se apreciaban las ideas de los alumnos. Algunas de estas cuestiones se ponían de manifiesto en los cuestionarios que diseñaron los alumnos para entrevistar a los profesores, así como en el análisis y reflexión sobre las respuestas.

A: “¿Cómo participamos en esta institución?, ¿Participamos de verdad?, ¿Quién controla las acciones del director?, ¿Qué funciones desempeña el Consejo Escolar?, ¿Cómo podrían participar los padres en la vida del centro?, ¿Cómo se gestionan los conflictos del centro?". [Cuaderno del alumno].

Esto implicaba una mayor reflexión sobre la convivencia en la escuela y ésta era inseparable de la reflexión sobre el propio proceso de aprendizaje, poniendo de manifiesto que eran conscientes de que, aunque había unos procedimientos de participación establecidos, en la práctica a menudo eran un mero formalismo, la convivencia en la escuela no contemplaba la participación efectiva de los alumnos.

En la reflexión sobre el propio proceso de aprendizaje, también se distinguía el papel de las partes (aprendiz-experto), los factores implicados (contenidos, metodología) y la complejidad de las relaciones. La metarreflexión tenía en cuenta el valor de las propias ideas y opiniones, y su cambio y evolución, demandando mayor participación en el propio proceso de aprendizaje. En cuanto a las metodologías y a los contenidos, ponían en cuestión su relevancia y criticaban un modelo basado exclusivamente en la memorización sin sentido de cosas que no consideraban útiles para su futuro.

Según ellos, la metáfora de la cárcel, que utilizaban continuamente, no sólo tenía que ver con espacios claustrofóbicos y con prohibiciones, sino también con las cosas que se hacen y cómo se hacen, con la falta de libertad, no sólo para moverse, también 
para opinar y pensar. En este sentido reclamaban unos nuevos contenidos, desde una concepción más compleja de lo que se debería enseñar en la escuela:

[La Escuela que queremos]: A: “Lo que estudiamos en la escuela y cómo lo hacemos: Que las clases sean más amenas y participativas, prácticas, divertidas y menos rutinarias y que los alumnos colaboren (...)”. [Cuaderno del alumno]

En definitiva, constatamos el paso de una concepción sobre el propio aprendizaje en la que predominaba la queja estéril por las deficiencias y los abusos a una reflexión más compleja, elaborada y simbólica, que tenía en cuenta distintos factores implicados en los procesos de aprendizaje. Y sobre todo aparecían propuestas en positivo para una escuela con actividades menos rutinarias y más participativas, donde "se nos tenga en cuenta en las decisiones que están relacionadas con nosotros".

\section{Conclusiones}

Finalmente, todo esto nos lleva a postular que la experimentación de un ámbito de investigación escolar sobre "El conflicto y la convivencia" contribuye en la práctica al aprendizaje de los alumnos, pues, aunque éste está condicionado por las representaciones sociales del alumnado y por la cultura escolar mayoritaria, son posibles reestructuraciones que den lugar a procesos de aprendizaje, entendiendo éstos como el enriquecimiento de los conocimientos previos (García Pérez, 2007).

Esta forma de trabajar revela, por tanto, potencialidades para educar en la ciudadanía participativa, pues desarrolla las destrezas relacionadas con la identificación y formulación de problemas sociales y su resolución, con la gestión del conflicto y la discrepancia en el trabajo en equipo, y con la reflexión crítica sobre el propio proceso de aprendizaje, que puede hacer realidad la tan citada competencia de "aprender a aprender", o lo que es lo mismo, la capacidad para seguir aprendiendo toda la vida.

En este sentido, confirmamos -en la línea de otras investigaciones-, como las revisadas por Fernández et al. (2006), o las realizadas en el marco del Proyecto IRES, que el modelo didáctico de aprendizaje basado en problemas, o de investigación en la escuela, (García Pérez, 2007) ofrece una alternativa válida para promover la reflexión, el debate y la construcción de conocimiento -con significado y sentido personal- sobre los fenómenos sociales. En esta alternativa, los conocimientos de los alumnos constituyen un referente fundamental para el ajuste de la intervención educativa según una determinada hipótesis de progresión de dicho conocimiento. Esto ha supuesto trabajar -en el proceso de investigación- en la construcción de un sistema de categorías metadisciplinares generales, en nuestro caso de unos metaprocedimientos, que funcionan como un andamiaje capaz de estructurar y organizar la comprensión de los fenómenos sociales y las actitudes y las habilidades procedimentales, propiciando un enriquecimiento y complejización del conocimiento de los alumnos (García Pérez, 2007). 


\section{Referencias}

Canal, M., Costa, D., \& Santisteban, A. (2012). El alumnado ante problemas sociales relevantes: ¿Cómo los interpreta? ¿Cómo piensa la participación? En N. de Alba, F. García, \& A. Santisteban (Eds.), Educar para la participación ciudadana en la enseñanza de las Ciencias Sociales (pp. 527-536). Sevilla, España: Díada.

Clark, P. (2013). Clio in the curriculum: Vindicated at last. Canadian Issues, (Summer), 42-46.

Cohen, L., Manion, L., \& Morrison, K. (2011). Research methods in Education. New York (NY), Estados Unidos: Routledge.

Danish Ministry of Education (2008). Executive Order on Upper Secondary School Leaving Examination. Recuperado de https://www.retsinformation.dk/Forms/ R0710. aspx?id=120566

Delval, J. (1994). El desarrollo humano. Madrid, España: Siglo XXI.

Fernández Martínez, M., García Sánchez, J. N., De Caso, A., Fidalgo, R., \& Arias, O. (2006). El aprendizaje basado en problemas: Revisión de estudios empíricos internacionales. Revista de Educación, 341, 397-418.

García, J. E. (1998). Hacia una teoría alternativa sobre los contenidos escolares. Sevilla, España: Díada.

García Pérez, F. F. (2007). El conocimiento cotidiano como referente del conocimiento escolar. En R. M. Ávila, R. Ruiz, E. López Atxurra, \& E. Fernández de Larrea (Eds.), Las competencias profesionales para la enseñanza-aprendizaje de las Ciencias Sociales ante el reto europeo y la globalización (pp. 481-498). Bilbao, España: Asociación Universitaria del Profesorado de Didáctica de las Ciencias Sociales.

García, F. F., \& De Alba, N. (2008). ¿Puede la escuela del siglo XXI educar a los ciudadanos y ciudadanas del siglo XXI? Scripta Nova. Revista Electrónica de Geografía y Ciencias Sociales, $270\left(\mathrm{n}^{\mathrm{o}}\right.$ extra 12), 122.

Gil, D., \& Vilches, A. (2006). Educación ciudadana y alfabetización científica: Mitos y realidades. Revista Iberoamericana de Educación, 42, 31-53.

González Cascón, E., De Juan, M. D., Parra Azor, J. F., Sarabia Sánchez, F. J., \& Kanther, A. (2010). Aprendizaje autorregulado: Antecedentes y aplicación a la docencia universitaria de marketing. Revista de Investigación Educativa, 28(1), 171-194.

González Ramírez, T., \& Correa Gutiérrez, S. (2006). Criterios e indicadores para la evaluación de procedimientos en el programa Ciencia y Tecnología para niños. Revista de Investigación Educativa, 24(1), 239-260.

González Cascón, E., De Juan, M. D., Parra Azor, J. F., Sarabia Sánchez, F. J., \& Kanther, A. (2010). Aprendizaje autorregulado: antecedentes y aplicación a la docencia universitaria de marketing. Revista de Investigación Educativa, 28(1), 171-194.

Goodson, I. (2000). El cambio en el currículum. Barcelona, España: Octaedro.

Heiman, G. W. (2011). Basic statistics for the behavioral sciences (6-a ed.). Belmont: Wadsworth Cengage Learning.

Hermann, R. S. (2008). Evolution as a controversial issue: A review of instructional approaches. Science and Education, 17(8-9), 1011-1032.

Jiménez-Aleixandre, M. P., \& Erduran, S. (2008). Argumentation in Science Education: An overview. In S. Erduran \& M. P. Jiménez-Aleixandre (Eds..), Argumentation in Science Education: Perspectives from classroom-based research (pp. 3-28). Berlín, Alemania: Springer. 
Johnson, D. W., \& Johnson, R. T. (2009). Energizing learning: The instructional power of conflict. Educational Researcher, 38(1), 37-51.

Krippendorff, K. (2013). Content analysis: An introduction to its methodology. Los Angeles (CA), Estados Unidos / Londres, Inglaterra: Sage.

McCoy, L. P. (Ed.) (2013). Studies in teaching 2013. Research Digest. Action research projects presented at Annual Research Forum. Winston-Salem (NC), Estados Unidos: Wake Forest University.

Millar, R. (1997). Science education for democracy: What can the school curriculum achieve. In R. Levinson \& J. Thomas (Eds.), Science today: Problem or crisis? (pp. 87-101). Londres, Inglaterra: Routledge.

Monereo, C. (Coord.) (2001). Ser estratégico y autónomo aprendiendo. Unidades de enseñanza estratégica para la ESO. Barcelona, España: Graó.

Nielsen, J. A. (2009). Structuring students' critical discussions through processes of decision-making on socio-scientific controversies. Revista de Estudio Universitarios, $35,139-165$.

Navarro, E., \& De Alba, N. (2011). El aprendizaje de la historia de España para la educación ciudadana. Investigación en la Escuela, 75, 21-34.

Oolbekkink-Marchand, H. M., Steen, J. van der, \& Nijveldt, M. (2014). A study of the quality of practitioner research in secondary education: Impact on teacher and school development. Educational Action Research, 22(1), 122-139. doi: 10.1080/09650792.2013.854175

Parker, W. C. (2008). Knowing and doing in democratic citizenship education. En L. S. Levstik \& C. A. Tyson (Eds.), Handbook of research in social studies education (pp. 65-80). Nueva York (NY), Estados Unidos/Londres, Inglaterra: Routledge.

Pineda-Alfonso, J. A., \& García-Pérez, F. F. (2011). La construcción de un ámbito de investigación escolar sobre el conflicto, la violencia y la guerra. Íber, Didáctica de las Ciencias Sociales, Geografía e Historia, 68, 29-36.

Qualification and Curriculum Authority (QUA) (2006). Key stage 4: Programme of study for science from 2006. Recuperado de http://www.qca.org.uk/ downloads/10340_ science_prog_of_study_from2006_ks4.pdf

Seixas, P., \& Peck, C. (2004). Teacher historical thinking. In A. Sears \& I. Wright (Eds.), Challenges and prospects for Canadian social studies (pp. 109-117). Vancouver, Canadá: Pacific Educational Press.

Siegler, R. S., \& Richards, D. D. (1989). El desarrollo de la inteligencia. In R. J. Sternberg (Ed.), Inteligencia humana: Evolución y desarrollo de la inteligencia (Vol. 4). Barcelona, España: Paidós.

Stradling, R. (1984). Controversial issues in the currículum. In R. Stradling, M. Noctor \& B. Baines (Eds.), Teaching controversial issues. Melbourne, Australia: Edward Arnold.

Young, M., Commings, E., \& Kington, C. (2002). Global citizenship: The handbook for Primary Teaching. Cambridge, Inglaterra: Chris Kington Publishing.

Fecha de recepción: 08/10/2014.

Fecha de revisión: 10/10/2014.

Fecha de aceptación: 21/03/2015. 
